\title{
Composition et rythme journalier de la dérive des exuvies nymphales de Chironomidés dans le Guadalquivir (Sierra de Cazorla - Espagne)
}

\author{
A. Vilchez-Quero' \\ P. Lavandier ${ }^{2}$
}

Mots clés : Chironomidae, exuvies nymphales, rythme journalier, dérive.

Les exuries nymphales et les imagos $\sigma$ de chironomidés obtenus par dérive au cours d'un cycle de 24 heures, en septcmbre 1985, ont permis de dénombrer 64 espèces: 10 sont nouvelles pour l'Espagne.

Les nombres d'espèces récoltées dans les échantillons horaires varient au cours de la journée : $\mathbf{4 2}$ sont récoltées au crépuscule, 23 à 36 la nuit et 15 à 25 seulement le matin.

Le cycle d'émergence de 13 espèces abondantes est étudié à partir des exuvies. Deux espèces émergent principalement le jour. Les autres émergent surtout au crépuscule et/ou durant la nuit. La réduction de la luminosité paraît constituer k facteur déterminant les émergences.

Composit ion and dlel periodicity of the drift of pupal exuviae of Chironomids in the river Guadalquivir (Slerra de Cazorla Spatn).

Keywords : Chironomidae, pupal exuviae, diel periodicity, drift.

Pupal exuviae and imagos ${ }^{\circ}$ of chironomids were collected hourly by drift netting, over 24 hours in september 1985 ; 64 species were identified, 10 of which are new to Spain.

The number of species showed a marked change between samples : 42 species were caught at sunset, 23 to 36 during the night and 15 to 25 in the morning.

The diel periodicity of emergence was studied by collecting exuviae for 13 abundant species. Only 2 species emerged primarily during day-light hours. The remainder emerged primarily at sunset and/or during the night and the reduction in light may be emergence cue.

\section{1. - Introduction}

Depuis Thienemann (1910), de nombreux auteurs comme Brundin (1949-1956), Lehman (1971) Coffman (1973), Ringe (1974), Mc Gill \& al (1979) Rossaro \& Ferrarese (1980), Wilson (1980), Laville (1979, 1981) ont utilisé les exuvies nymphales capturées par dérive pour étudier les peuplements de chironomidés des eaux courantes. En effet cette méthode simple résout pratiquement les problèmes liés à l'hétérogénéité du milieu (Mc Gill \& al 1979), au caractère aléatoire de la capture des imagos et aux difficultés

1. Departamento Biología Animal y Ecología, Facultad de Cien(ias, Universidad de Granada, Espana.

2. Laboratoire d'Hydrobiologie, UA 695 C.N.R.S. Université Paul Sabatier, 118 route de Narbonne, 31062 Toulouse Cédex, France. d'identification du matériel larvaire. Les relevés renseignent également sur la phénologie des espèces (Coffman 1973 et 1974, Wartinbee 1979, Rieradevall 1985) et sont en outre suffisamment riches et représentatifs (Wilson \& Bright 1973) pour limiter l'effort d'échantillonnage : sur la rivière Lot, Laville (1981) note que deux séries de prélèvements suffisent pour récolter $90 \%$ des espèces et Wilson (1980) estime qu'à défaut de relevés répartis sur l'année un unique prélèvement estival, suffisamment conséquent, permet d'établir une liste d'espèce valable.

Dans ce travail, nous étudions les exuvies nymphales et les imagos de Chironomidés capturés par dérive au cours d'un cycle de 24 h dans le cours supérieur du Guadalquivir dans le but $1^{\circ}$ de dresser 


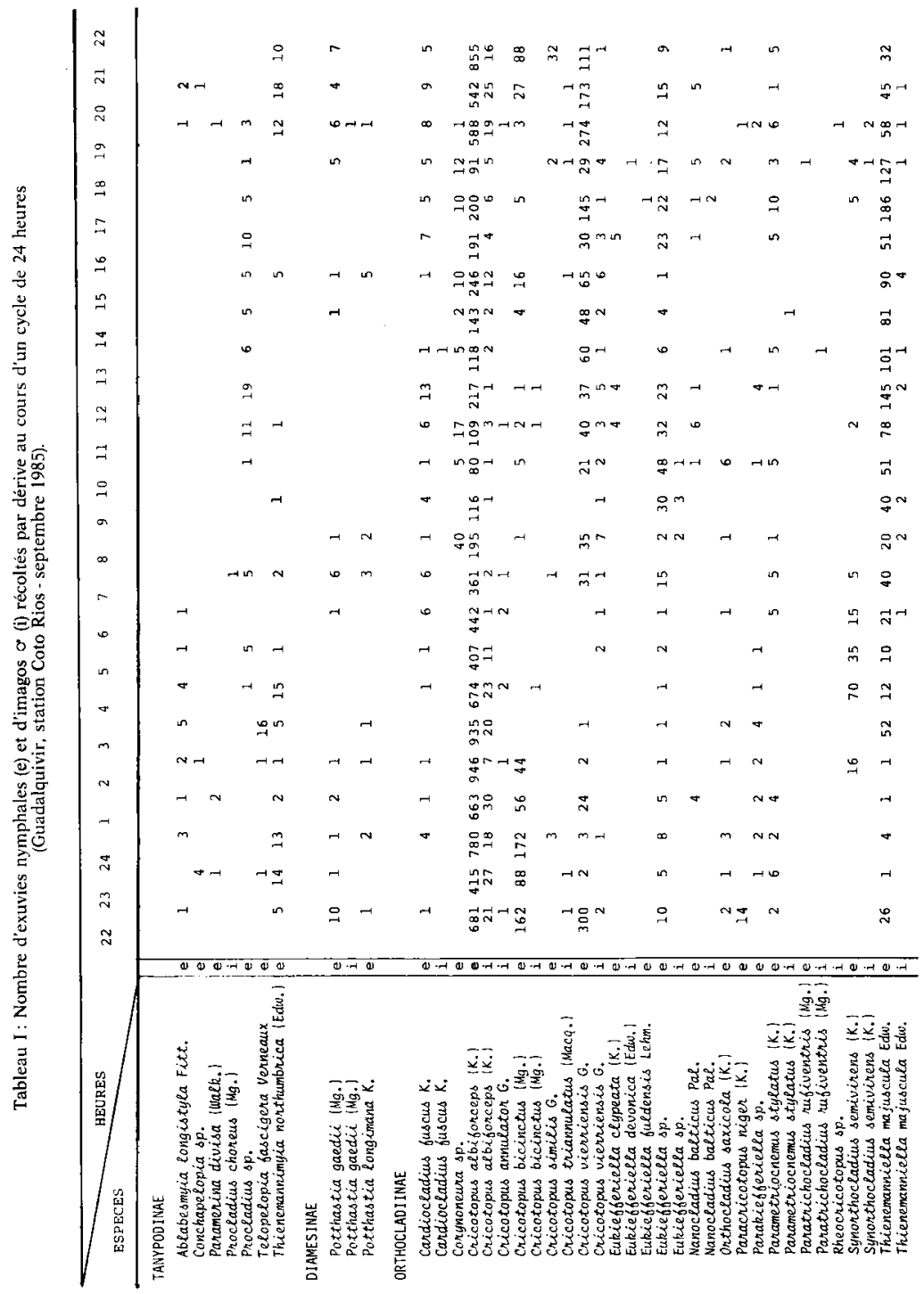




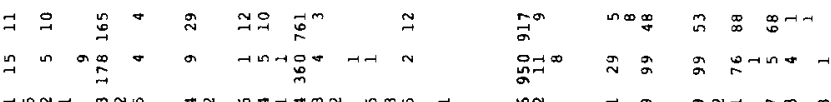

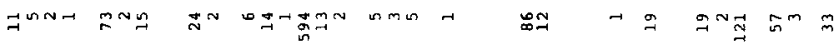

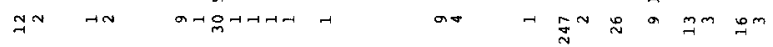

$$
\begin{aligned}
& \text { m H }
\end{aligned}
$$

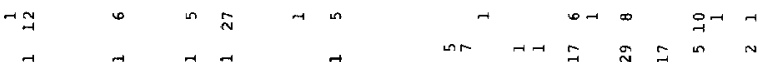

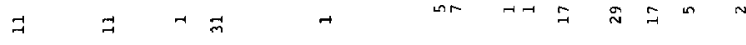

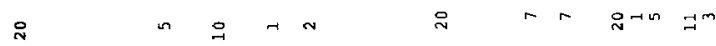

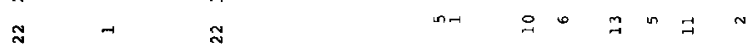

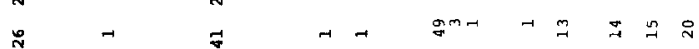

$$
\begin{aligned}
& \text { I } \rightarrow \text { N } \rightarrow \text { or } \rightarrow \text { or or inmo in } \\
& \Rightarrow \text { m N } \rightarrow \text { in } \rightarrow m \text { i } \\
& \text { - }
\end{aligned}
$$

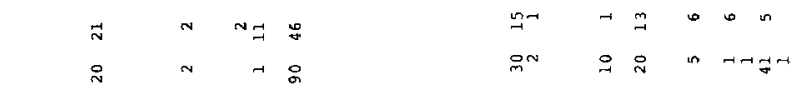

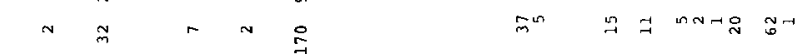

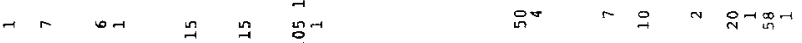

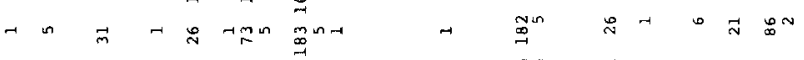

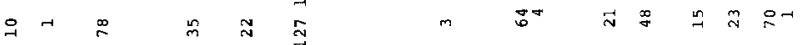

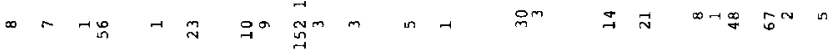

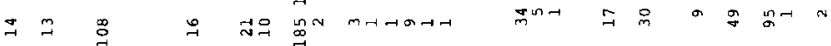

$$
\begin{aligned}
& \text { v } \Rightarrow \text { - }
\end{aligned}
$$

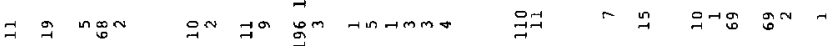

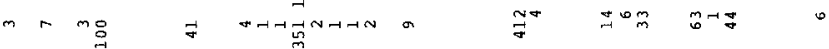

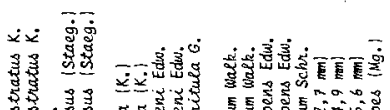

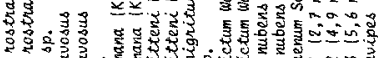

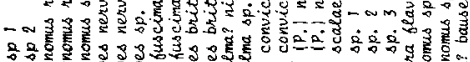

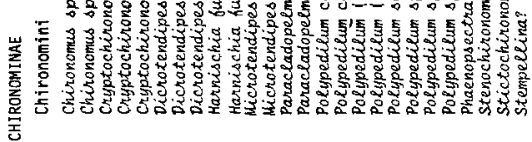

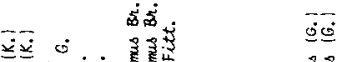

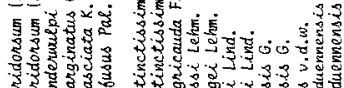

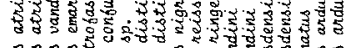

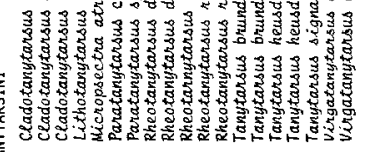


un premier inventaire des Chironomidés de cette région d'Espagne (Sierra de Cazorla), $2^{\circ}$ de comparer la valeur faunistique des dérives de courte durée effectuées à des heures différentes, $3^{\circ}$ de préciser le rythme nycthéméral d'émergence des espèces dominantes à partir de l'évolution numérique des exuvies nymphales.

\section{2. - Station d'étude et conditions d'expérience}

La station - Coto Rios - est située sur le Guadalqui$\operatorname{vir}$ ( $30 \mathrm{~km}$ de la source $-680 \mathrm{~m}$ d'altitude) $200 \mathrm{~m}$ en aval d'une petite retenue. A ce niveau. le cours est large de 10 à $12 \mathrm{~m}$, profond de 15 à $30 \mathrm{~cm}$ avec un substrat de pierres et de sable grossier.

Les prélèvements de dérive ont été réalisés du $11.09 .1985,15 \mathrm{~h}$ au 12.09.1985, $15 \mathrm{~h}$. Le filet de dérive (ouverture rectangulaire $25 \times 35 \mathrm{~cm}$ - vide de maille $250 \mu \mathrm{m})$ reposait sur le fond et filtrait toute la colonne d'eau. Il était relevé toutes les heures. Au cours de ce cycle de 24 h, le ciel est resté dégagé et le débit constant avec une vitesse de courant d'environ $30 \mathrm{~cm} \mathrm{~s}^{-1}$ à l'entrée du filet ; la température de l'eau a varié de $18,5(7 \mathrm{~h})$ à $22,5^{\circ} \mathrm{C}(16 \mathrm{~h})$. Le matériel obtenu est fixé au formol à $4 \%$, trié sous loupe binoculaire et conservé en alcool $70 \%$.

\section{3. - Résultats}

\section{1. - Composition faunistique}

26631 exuvies nymphales et 562 imagos ơ ont été récoltés et identifiés (Tableau I). Ils appartiennent à 64 espèces dont 10 sont citées pour la première fois en Espagne (cf. 3.2.). Les Orthocladiinae et les Chironomini (20 espèces chacun) sont plus diversifiés que les Tanytarsini (14 espèces), les Tanypodinae (6 espèces) et les Diamesinae (2 espèces).

Du point de vue numérique, les Orthocladiinae représentent $53,2 \%$ des récoltes d'exuvies avec 4 espèces principales Cricotopus albiforceps $37 \%, C$. vierriensis (5\%), C. bicinctus $(2,5 \%)$ et Thienemanniella majuscula $(4,8 \%)$ identifiées à partir de nymphes mâles. Les Chironominae représentent $45,5 \%$ des récoltes réparties à part égale entre les Chironomini $22 \%$ et les Tanytarsini $(23,4 \%)$. Deux espèces dominent nettement cette communauté Polypedilum nubens (7\%) et Cladotanytarsus atridorsum (12\%). Plus de la moitié des espèces présentent une abondance inférieure à $0,1 \%$ de l'effectif total capturé.
Parmi les 30 espèces d'imagos recueillies, 7 (Cricotopus annulator, C. triannulatus, Eukiefferiella devonica, Rheo cricotopus sp., Polypedilum scalaenum, Cladotanytarsus vanderwulpi et Polypedilum signatus n'avaient pas été récoltées à l'état exuvial. Les quatre espèces les plus abondantes Cricotopus albiforceps $(45,7 \%)$ C. vierriensis $(7,6 \%)$ Polypedilum (P) nubens (7\%) et Cladotanytarsus atridor. stum (17,8\%) qui dominaient également parmi les exuvies peuvent caractériser la communauté des Chironomidés à la station Coto Rios.

\section{2. - Distribution des espèces nouvelles pour l'Espagne}

\section{TANYPODINAE}

Paramerina divisa (Walk). Espèce sténotherme d'eau froide et polyoxybionte qui colonise les milieux courants et la zone littorale des lacs (Fittkau 1962, Ferrarese 1983).

Telopelopia fascigera Verneaux. Espèce seulement connue de France (Laville \& Viaud-Chauvet 1983). Fréquente dans les grands cours d'eau a forte amplitude thermique annuelle et à degré élevé de trophie (Verneaux 1970). Elle peut caractériser le potamal (Laville 1981). Rare dans nos récoltes.

\section{ORTHOCLADIINAE}

Cricotopus (C) albiforceps (K.). Ce Cricotopus du groupe festivellus, surtout signalé en faciès lentique (Hirvenoja 1973. Fittkau \& Reiss 1978) peuple aussi les milieux cou rants comme les rivières Truyère et Lot (Laville 1979). A Rio Cotos c'est le Chironomidé le plus abondant.

Eukiefferiella fuldensis Lehm. Espèce connue des zones supérieures des cours d'eau : épirhithral de la Fulda (Lehmann 1971), torrent d'Estaragne dans les Pyrénées (Laville \& Lavandier 1977). Récoltée dans la rivière Touyre (Brouquet-Laglaire 1985). Dans le Massif Central, signalée dans le crénal et l'épirhithral du Lot (Laville 1981), dans l'épi et le métapotamal de l'Aveyron (Viaud-Chauvet 1982) et dans la rivière Dadou (Brouquet-Laglaire 1985). Rare à la station prospectée dans le Guadalquivir.

Nanocladius balticus Pal. Espèce connue des lacs vaseux eutrophes (Fjttkau \& Lehmann 1970). Elle est plus rarement récoltée au confluent des rivières Lot et Truyère (Laville 1979), dans le potamal de l'Aveyron (Viaud-Chauvet 1982) et du Lot (Brouquet-Laglaire 1985). Rare dans nos récoltes.

Thienemanniella majuscula Edw. Uniquement connue des îles britanniques (Fittkau \& Reiss 1978) où elle est signalée des rivières (Langton 1984). Fréquente à la station avec près de $5 \%$ des captures.

\section{ChironominaE}

\section{Chironomini}

Microtendipes britteni Edw. Ce Microtendipes colonise plus particulièrement les zones littorales des lacs 
(Thienemann 1950. Laville 1971). Signalée également dans les cours moyen et supérieur d'un ruisseau du Mittelge. birge entre $556 \mathrm{~m}$ et $625 \mathrm{~m}$ (Dittmar 1955). Fréquente dans nos relevés.

Paracladopelma nignitula G. Cette espèce vit dans la zone littorale des lacs oligotrophes (Jackson 1977, Langton 1984). Espèce rare à la station étudiée.

\section{Tanytarsini}

Cladotanytarsus atridorsum $\mathbf{K}$. Espèce lénitique des lacs et réservoirs (Langton 1984). Rare dans la partie haute de la rivière Lot (Laville 1981). Abondante dans nos récoltes elle provient de la retenue.

Lithotanytarsus emarginatus (G.). Signalée dans les Pyré. nées espagnoles (Bertrand 1952, 1953 et 1956). Espèce caractéristique des eaux calcaires (Thienemann 1934) ce qui justifie sa présence dans la Sierra de Cazorla : Margalef et al (1976) on noté une concentration en $\mathrm{Ca}^{+}+$voisine de $100 \mathrm{mg} \mathrm{1}^{-1}$ dans le réservoir du Tranco de Beas où se jette le Guadalquivir, $10 \mathrm{~km}$ en aval de la station de Coto Rios.

Rheotanytarsus distinctissimus $\mathrm{Br}$. Cette espèce paraît être une forme d'eau courante (Lehmann 1970). Signalée des rivières Lissuraga dans les Pyrénées atlantiques (Laville 1980), et Lot dans le Massif Central (Laville 1981). Son abondance dans le Guadalquivir (2,7\%) confirme sa tendance rhithrobionte.

\subsection{Caractéristiques de la dérive au cours de la journée}

Le nombre d'espèces et le nombre d'individus suivent la même évolution générale, caractérisée par la prépondérance des valeurs nocturnes (fig. 1).

Les récoltes les plus diversifiées sont faites au crépuscule : 42 espèces capturées entre 19 et $20 \mathrm{~h}$ soit $66 \%$ du nombre total d'espèces obtenues au cours

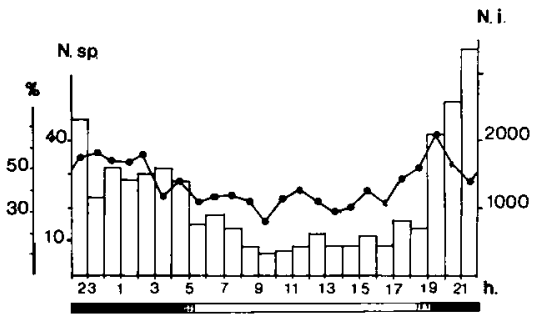

Fig. 1 : Variation du nombre d'individus ( $\mathrm{Ni}_{\text {. }}$ - diagramme) et du nombre d'especes (N.sp. - courbe) capturés par heure au cours d'un cycle de 24 heures. Le nombre d'es. pèces est également exprimé en \% du nombre total d'espèces récoltées. du cycle de $\mathbf{2 4}$ heures. Les relevés nocturnes (avec en moyenne 32 espèces différentes récoltées entre $20 \mathrm{~h}$ et $4 \mathrm{~h}$ ) sont nettement plus riches que les relevés diurnes qui renferment en moyenne 22 espèces.

Le minimum - 15 espèces soit $23 \%$ du total - est observé entre $9 \mathrm{~h}$ et $10 \mathrm{~h}$ du matin. Sur un total de 64 espèces, 41 sont absentes dans plus de la moitié des relevés; en revanche, 13 des 14 espèces dominantes dont l'abondance est voisine ou supérieure à $1 \%$ des effectifs totaux figurent dans 20 relevés au moins.

L'évolution numérique des exuvies qui reflète le cycle nycthéméral d'émergence des espèces dépend essentiellement des formes principales. Quatre types de dérive peuvent être reconnus parmi les 13 espèces dominantes ( $f$ ig. 2).

- La dérive est essentiellement diurne avec 2 maxima le matin et l'après-midi (Thienemanniella majuscula, Eukiefferiella sp.);

- La dérive présente un maximum au crépuscule (Rheotanytarsus distinctissimus, Tanytarsus brundini) ou dans les premières heures de la nuit (Dicrotendipes nervosus, Rheotanytarsus reissi, Cricotopus vierriensis, Cladotanytarsus atridorsum. Polypedilum nubens).

- La dérive présente deux maxima en début et en fin de nuit (Microtendipes britteni);

- La dérive est surtout nocturne sans maximum net (Tanytarsus heusdensis, Cricotopus albiforceps, Cricotopus bicinctus).

Les autres espèces, moins abondantes, dérivent préférentiellement la nuit.

\section{4. - Discussion}

Parmi les 62 espèces recensées, 10 sont citées pour la première fois en Espagne: Paramerina divisa, Telopelopia fascigera, Cricotopus (C.) albiforceps, Eukiefferiella fuldensis, Nanocladius balticus, Thienemanniella majuscula, Microtendipes britteni, Paracladopelma? nigritula, Cladotanytarsus alridorsum et Rheotanytarsus distinctissimus.

Deut d'entre elles, Cricotopus (C.) albiforceps et Cladotanytarsus atridorsum peuvent définir avec Cricolopus vierriensis et Polypedilum nubens la communauté des chironomidés de cette zone du Guadalquivir assimilable à un hyporhithral. La faune qui regroupe des formes de faciès lotique et lentique 

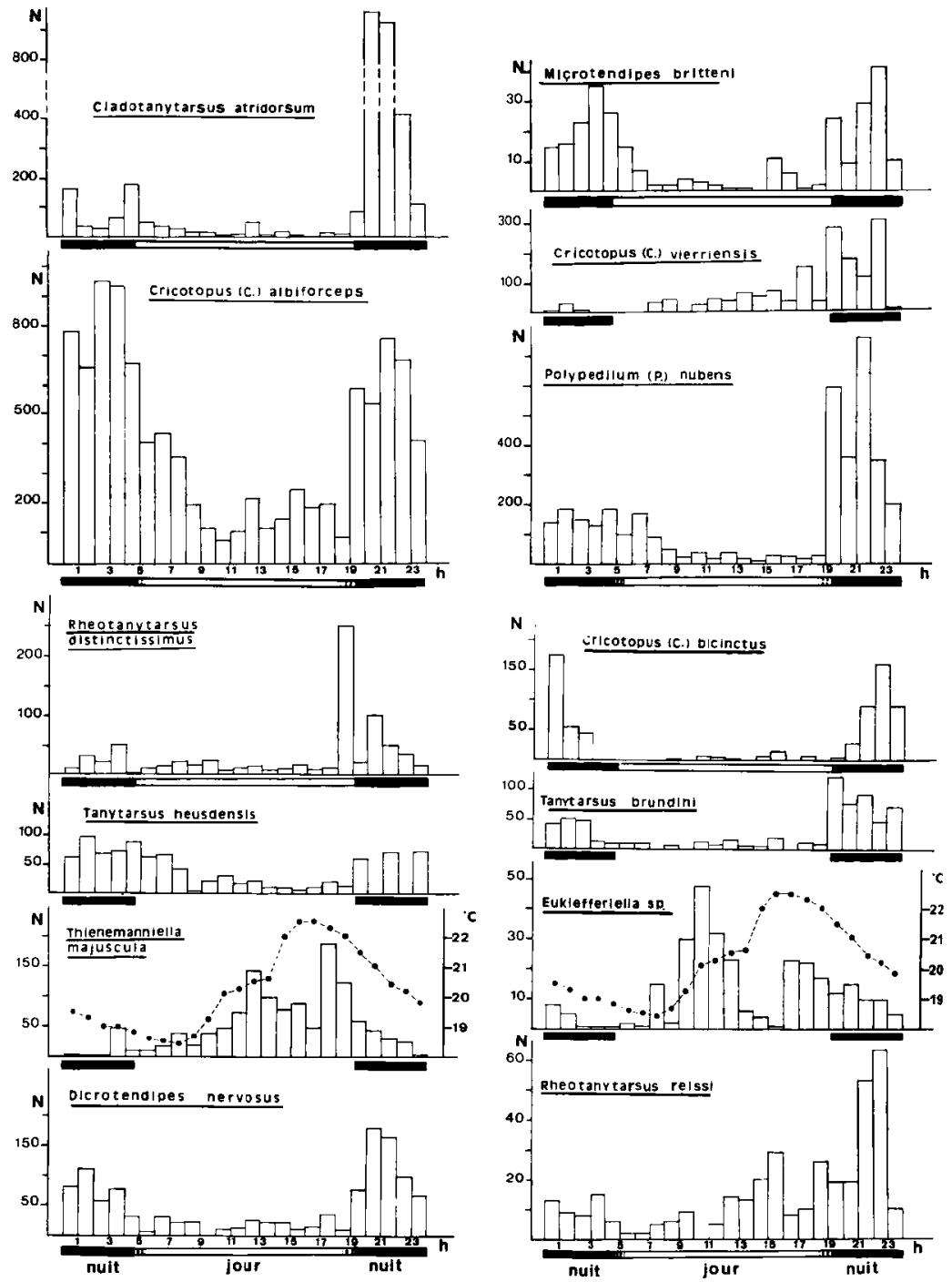

Fig. 2 : Rythme quotidien de dérive des exuvies nymphales des espèces principales. $\mathrm{N}:$ nombre d'exuvies, $\mathrm{h}$ : heures solaires 
reflète bien la situation de la station et plus généralement la modification des milieux d'eau courante consécutive à leur aménagement (Laville \& ViaudChauvet 1985). La pauvreté des récoltes en Diamesinae et Tanypodinae s'explique, pour les premiers par la faible altitude de la station et pour les seconds par la migration des larves vers les bords avant la nymphose.

Les relevés horaires n'apportent pas tous la même information, chacun d'eux sous-estimant en outre assez largement le nombre total d'espèces présentes dans le milieu. Dans le Guadalquivir, le relevé le plus diversifié, fait au crépuscule, regroupe seulement $2 / 3$ des espèces capturées au cours du cycle de $24 \mathrm{~h}$; le relevé le moins diversifié, fait le matin, en recueille moins du quart - toutes les espèces principales sont néanmoins présentes. Ces proportions, relativement faibles, concordent avec celles obtenues dans le Llobregat (Rieradevall 1985) où 1 heure de dérive apporte entre 12 et $70 \%$ des espèces récoltées sur $24 \mathrm{~h}$.

Si on allonge la durée des prélèvements de dérive, le nombre d'espèces présentes dans chacun d'eux augmente sensiblement. Ainsi, selon les périodes considerées, un relevé de 2 heures de dérive consécutives apporte 39 à $78 \%$ des espèces ; un relevé de 3 heures de dérive consécutives en recueille 43 à $81 \%$. En pratique toutefois, il est difficile d'allon. ger la durée des dérives (colmatage du filet - temps nécessaire au dépouillement des données) et il est préférable de cumuler plusieurs prélèvements de courte durée bien répartis au cours de la journée. La nuit constitue toujours une période privilégiée mais le protocole d'échantillonnage doit être adapté à chaque situation : dans le Llobregat par exemple, les échantillons les plus diversifiés sont faits à l'aube (Rieradevall 1985). D'autre part, il est vraisemblable que dans les rivières importantes, des relevés de courte durée donnent des résultats relativement homogènes compte tenu de la durée de flottaison des exuvies (Wilson et Bright 1973, Coffman 1973) et des conditions d'écoulement.

Les prélèvements de dérive peuvent être utilisés pour appréhender le rythme des émergences (Wartinbee 1979, Rieradeval1 1985) même si la durée du transport étale dans le temps les récoltes. Ainsi dans nos prélèvements, les exuvies sont recueillies sur des périodes plus longues que les imagos et leurs variations numériques sont moins accusées que celles que l'on peut observer avec des nymphes ou des adultes piégés à l'émergence (Palmén 1955, 1956).

Dans le Guadalquivir, les positions relatives du filet et de la retenue tendent à retarder d'environ 1/2 heure la manifestation de l'émergence des espèces lentiques et à étaler dans le temps les récoltes. Cladotanytarsus atridorsum et Tanytarsus heusdensis présentent donc des émergences plus strictement nocturnes que ne le laissent supposer les récoltes d'exuvies ; elles se comportent comme en Finlande où elles émergent durant la brève période nocturne (Palmén 1955). Les conditions d'écoulement pourraient expliquer en partie les différences observées dans les dérives de Cricotopus bicinctus et Tanytarsus brindini qui sont beaucoup plus nocturnes dans le Guadalquivir que dans le Llobregat (Rieradevall 1985).

Dans le Guadalquivir, la majorité des émergences a lieu au crépuscule et dans les 4 heures suivantes. La réduction de la luminoșité dont l'incidence a été soulignée par Palmén (1955) et Morgan \& Waddel (1961) constitue certainement le facteur prépondérant dans le déterminisme des émergences. En revanche, le lever du soleil n'a guère d'influence, excepté sur Microțendipes britteni qui réagit nettement à tout changement de luminosité. Les variations de température qui sont parfois évoquées pour expliquer le rythme des émergences (Danks \& Oliver 1972, Wartinbee 1979) n'ont pas ici de rôle apparent ; même chez les espèces à dérive essentiellement diurne, il n'existe pas de relation nette entre la quantité d'exuvies recueillies et l'évolution quotidienne des températures.

\section{Remerciements}

Nous remercions le $\mathrm{D}^{r} \mathrm{H}$. Laville (Toulouse) pour avoir bien voulu confirmer la détermination de certaines especes.

\section{Travaux cités}

Bertrand (H.), 1952. - Récoltes de Diptères Chironomides dans les Pyrénées (2c note), Vie el Milieu, 3 (3) : 314-321.

Bertrand (H.), 1953. - Diptères Chironomides pyrénéens (2* note), Bull. Soc. ent. Fr., 58: 76.79.

Bertrand ( $\mathrm{H}$.). 1956. - Diptères Chironomides pyrénéens et espagnols, Bull. Soc. ent. Fr. $61: 93-95$.

Brouquet-Laglaire (Y.). 1985. - Etude des Chironomidés (Diptera) de quelques rivières polluées du bassin de la Garonne : Touyre, Agout, Dadou, Thoré, bas Lot, Thèse 3' cycle, Toulouse : $237 \mathrm{p}$. 
Brundin (L.). 1949. - Chironomiden und andere Bodentiere der sudschwedischen Urgehirgseen, Inst. Freshwater Res. Drottningholm, $30: 1-194$.

Brundin (L.). 1956. - Zur Systematik der Orthocladiinae (Dip. Chironomidae), Rep. Inst. Freshwater Res. Drottningholm, 37 : 5-185.

Coffman (W.P.) 1973. - Energy flow in a woodland stream ecosvstem : II. The taxonomic composition and phenology of the chironomidae as determined by the collection of pupal exuviae, Arch. Hydrobiol, 71 : 3, 281-322.

Coffman (W.P.). 1974. - Seasonal differences in the diel emergence of a lotic chironomid community, Ent. Tidskr. Suppl., 95 : $42-48$.

Danks (H.V.) \& Oliver (D.R.). 1972. - Seasonal emergence of some high arctic Chironomidae (Diptera), Can. Entomol., 104: $661-686$

Dittmar (H.). 1955. - Ein Sauerlandbach, Arch. Hydrobiol., 50 : $305-552$.

Ferrarese (U.). 1983. - Guide per il riconoscimento delle specie animali delle acque interne italiane. 26. Chinonomidi, 3 - (Diptera : Chironomidae : Tanypodinae), $\mathrm{AQ} / 1 / 204$. Consiglio nazionale delle ricerche. Coordinatore: S. Ruffo, Verona., $67 \mathrm{p}$.

Fittkau (E.J.). 1962. - Die Tanypodinae (Dipt. Chironomidae). Abh. Larvalsyst. Insekten, 6: 1-453.

Fittkau (E.J.) \& Lehmann (J.). 1970. - Revision der Gattung Microcricotopus Thien, u. Harn. (Dipt. Chironomidae), Imt. Revue ges. Hydrobiol. Hydrogr., 55 (3); 391-402.

Fittkau (E.J.) \& Reiss (F.). 1978. - Chironomidae. In Illies J., éd. Limnofauna Europaea : 404440, G. Fischer, Stuttgart.

Hivernoja (M.). 1973. - Revision der Gattung Cricolopus van der Wulp und ihrer Verwandten (Diptera, Chironomidae), Ann. Zool. tenn., 10:1-363.

Jackson (G.A.). 1977. - Nearctic and Palearctic Paracladopelma Harnich and Saetheria n. gen. (Diptera : Chironomidae), J. Fish. Res. Buard Can., 34 : 1322.1359.

Langton (R.H.). 1984. - A key to plopal exuviae of British Chirono midae : 324 p. P.H. Langton (Ed.) March, Cambridgeshire, Great Britain.

Laville (H.) 1971. - Recherches écologiques sur les Chironomides (Diptera) lacustres du Massif de Néouvielle (HautesPyrénés). Première partie : systématique, écologie, phénologie, Ammls Limnol., 7 (2) : 173-332.

Laville (H.). 1979. - Etude de la dérive des exuvies nymphales de Chironomides au niveau du confluent Lot-Truyère, Annls. Limnot., 15 (2) : $155-180$

Laville (H.). 1980. - Inventaire 1980 des Chironomides (Diptera) connus des Pyrénées, Annls. Limnol. 16:211-223.

Laville (H.). 1981. - Récoltes des exuvies nymphales de Chironomides (Diptera) dans le Haut-Lot, de la source (1 $295 \mathrm{~m}$ ), au confluent de la Truyère $(223 \mathrm{~m})$, Annls. Limnol., $17: 225.289$.

Laville (H.) \& Lavandier (P.). 1977. - Les Chironomides (Diptera) d'un torrent pyrénéen de haute montagne : l'Estaragne. Annls. Limnol., $13: 57.81$.

Laville (H.) \& Viaud-Chauvet (M.). 1983. - Description de la nymphe de Telopelopio fascigera (Verneaux) (Diptera, Chironomidae). Annls. Limnol., 19 (1): 25-28.

Laville (H.) \& Viaud-Chauvet (M.). 1985. - Etude comparée de la structure des peuplements de Chironomidés dans cing rivières du Massif Central : relation entre cette structure et la qualité des eaux, Verh. Intemat. Verein. limnol, 22 : 2261.2269.

Lehmann (J). 1970. - Revision der europaischen Arten (Imagines $\sigma \sigma$ und Puppen $\sigma \sigma$ ) der Gattung Rheotanytarstus Bause (Diptera, Chironomidae), Zool. Anz., $185: 345-378$.
Lehmann (J.). 1971. - Die Chironomiden der Fulda (Systematische, okologische und faunistiche Untersuchungen). Arch. Hydrobiol. Suppl., 37 : 466-555.

Margalef (R.), Planas (D.), Armengol (J.), Vidal (A.), Prat (N.), Guiset (A.). Toja (J.) \& Estrada (M.). 1976. - Limnologia de los embalses espanoles. Dirección General de Obras Hidraúlicas Ministerio de Obras Públicas. Madrid, 422 p.

Mc Gill (J.D.), Wilson (R.S.) \& Brake (A.M.). 1979. - The use of Chironomid popul exuviac in the surveillance of sewage pollution within a drainage system, Water Research., 13:887-894.

Morgan (N.C.) \& Waddell (A.B.). 1961. - Diurnal variation in the emergence of some aquatic insects, T. Roy. Ent. Soc. Lond. 113 123-137.

Palmén (E.). 1955. - Diel periodicity of pupal emergence in natu. ral populations of some chironomids, Ann. Zool. Soc "Wanamon, $17: 1.30$

Palmén (E.) 1956. - Periodic emergence in some chironomids and adaptation to nocturnalism, Festskr, för Hanström. Lund. 248-256.

Rossaro (B.) \& Ferrarese (V.). 1980 - A contribution to the knowledge of Chironomids in Italy, including cluster analysis of presence absence data and factor analysis with per cent composition of species in a stream. In Chironomidae, Ecology, Systematics, Cytology and Physiology. Murray D.A., ed., Pergamon Press, Oxford : 203-208

Rieradevall (M.) 1985 - Ritme diari de la deriva en una estacio dcl riu Llobregat : Amb especial atencio a les exuvies pupals dels Chironomidae (Dipt.). Tesi de Licenciatura, Univ. Barcelona: $175 \mathrm{p}$

Ringe (F.) 1974. - Chironomiden-Emergenz 1970 in Breitenbach und Rohrwienbach. Schlitzer Produktions-biologische Studien 10. Arch. Hydrobiol. Suppl., $45: 212.304$.

Thienemann (A.). 1910. - Das Sammeln von Puppenhauten der Chjronomiden. Eine Bitte um Mittarbeit, Arch. Hydrobiol, 6: 213-214.

Thienemann (A). 1934. - Eine Gesteinsbildende Chironomidae. lithotanytarsus emarginsatus (Gothgebuer), Z. Morph. u. Okol. d. Tiere $28: \mathbf{4 8 0 - 4 9 6}$.

Thienemann (A.). 1950. - Lunzer Chiroromiden, Arch. Hydrobiol. 18: $\{-202$

Thienemann (A.). 1954. - Chironomus. Leben, Verbreitung und wirschafliche Bedeutung der Chironomiden, Binnengewässer., $20: 834 \mathrm{p}$

Verneaux (J.) 1970. - Telopelopia tascigera n. sp. une nouvelle espèce de Tanypodinae (Diptera, Chironomidae). Description de l'imago $\sigma$ et données écologiques, Ann. Scient. Univ. Besançon, Zool., $6: 59.64$

Viaud.Chauvet (M.) 1982. - Analyse des récoltes d'exuvies nymphales de Chironomides dans les rivières du bassin du Tam, Thèse 3c cycle. Ecologje. U.P.S. Toulouse : 171 p.

Wartinbee (D.C.). 1979, - Diel emergence patterns of lotic Chironomidae. Freshwater Biol., 9 : 147.156.

Wilson (R.S.) 1980. - Classifying rivers using Chironomid pupal exuviae. In Chironomidae, Ecology, Systematics, Cylology ans Physiology. Murray D.A., ed, Pergamon Press, Oxford ans New York : 209-216.

Wilson (R.S.) \& Bright (P.L.). 1973. - The use of Chironomid pupal exuviae for characterizing strearns, Freshwat. Biol. $3: 283$-302.

Wilson (R.S.) \& McGill (J.D.). 1977. - A new method of monotoring water quality in a stream receiving sewage effluent, using Chironomid pupal exuviae, Water Research, 11:959-962. 